\title{
Contemporary treatment of chronic osteomyelitis: implementation in low- and middle-income countries
}

\author{
Geurts $\mathrm{J}^{1}$, Vranken T², Gabriels F³, Arts $\mathrm{JJ}^{4}$, Moh P5 \\ ${ }^{1}$ MD; Attending Orthopaedic Surgeon, Department of Orthopaedic Surgery, CAPHRI School of Public Health and Primary Care, Maastricht University, \\ Maastricht, The Netherlands \\ 2 MSc; Medical Student, Department of Orthopaedic Surgery, CAPHRI School of Public Health and Primary Care, Maastricht University, Maastricht, The \\ Netherlands \\ ${ }^{3}$ MSc; Department of Orthopaedic Surgery, CAPHRI School of Public Health and Primary Care, Maastricht University, Maastricht, The Netherlands; \\ Orthopaedic Biomechanics Group, Department of Biomedical Engineering, Eindhoven University of Technology (TU/e), The Netherlands \\ 4 PhD; Department of Orthopaedic Surgery, CAPHRI School of Public Health and Primary Care, Maastricht University, Maastricht, The Netherlands; \\ Associate Professor, Orthopaedic Biomechanics Group, Department of Biomedical Engineering, Eindhoven University of Technology (TU/e), The \\ Netherlands \\ ${ }^{5} \mathrm{MD}$; Attending Orthopaedic Surgeon, St John of God Hospital, Duayaw Nkwanta, Ghana
}

Corresponding author: Dr J Geurts, Department of Orthopaedics, Maastricht University Medical Centre, PO Box 5800, 6202 AZ Maastricht, The Netherlands; tel +31-43-3875038; email: j.geurts@mumc.nl

\begin{abstract}
Aim: Chronic osteomyelitis is still a difficult problem to treat in the developed world, but even more so in low- and middle-income countries. Contemporary treatment options result in satisfying outcomes in a setting with abundant resources, but the question is whether these treatment options can be translated to other, less supported health care systems and if they obtain the same results.

Methods: Eighteen patients with established chronic osteomyelitis (eight type III, ten type IV) were prospectively enrolled and treated in a one-stage procedure with radical debridement and dead space management using bioactive glass S53P4 granules, together with adjuvant antibiotic therapy.

Results: Thirteen patients were assessed at 24 months. Infection control was achieved in five patients (38\%). Eight patients (61.5\%) had persistence or recurrence of infection. Loss to follow-up was substantial (five patients, 28\%).

Conclusion: Due to specific challenges treating chronic osteomyelitis in low- and middle-income countries, contemporary treatment options cannot be 'copy-pasted' with the same results in these settings.
\end{abstract}

Level of evidence: Level 4

Key words: osteomyelitis, bio-active glass, biomaterial, low and middle-income countries

Citation: Geurts J, Vranken T, Gabriels F, Arts JJ, Moh P. Contemporary treatment of chronic osteomyelitis: implementation in low- and middle-income countries. SA Orthop J 2018;17(2):40-43. http://dx.doi.org/10.17159/2309-8309/2018/v17n2a7

Editor: Dr LC Marais, University of KwaZulu-Natal
Received: July 2017
Accepted: November 2017
Published: May 2018

Copyright: @ 2018 Geurts J, et al. This is an open-access article distributed under the terms of the Creative Commons Attribution Licence, which permits unrestricted use, distribution and reproduction in any medium, provided the original author and source are credited.

Funding: The following benefits have been received from a commercial party: Bonalive® 1.0-2.0 mm granules, provided in kind by BonAlive Biomaterials Ltd, Turku, Finland.

Conflict of interest: None to report. 


\section{Introduction}

Chronic osteomyelitis is still a difficult problem to treat in the developed world, but even more so in developing, low- and middleincome countries (LMICs). Musculoskeletal infections in general can be the reason for hospital admission in as much as $14.5 \%$ of cases in these countries. ${ }^{1}$ Contemporary treatment options, such as the use of bioactive glass in a one-stage setting, result in satisfying outcomes in a setting with abundant resources, but the question is whether these treatment options can be translated to other, less supported health care systems and if they obtain the same results. Specific challenges come particularly with the treatment of chronic osteomyelitis in the setting of low- and middle-income countries: lack of good diagnostic tools (imaging as well as microbiology), availability of proper antibiotics and the possibility of administering these intravenously and for the proper length of time, conditions of surgery and adequate follow-up possibilities. ${ }^{2}$ This often results in misdiagnosis and/or under-treatment. ${ }^{3}$ Treatment often requires long hospitalisation which can lead to financial problems for the affected patients and their families as well as the health care system of the country concerned. Recurrence rates in the most ideal conditions can still be around 30\%, but are of unknown magnitude in LMICs. ${ }^{4}$ The aim of this study was to evaluate if a favourable outcome could be obtained using a treatment protocol from a European dedicated infection unit (Maastricht University Medical Centre, the Netherlands) in a setting with much fewer resources.

\section{Patients and methods}

A series of 18 consecutive patients with chronic osteomyelitis was treated in Ghana over a two-week period in March 2014. This occurred in a 150-bed district hospital which serves as a local referral centre for orthopaedic surgery. X-ray and ultrasound services are also provided. All patients were diagnosed with chronic osteomyelitis, half of which were post-traumatic in origin. The group included 15 men and three women. The average age was 26 years (range 10-70 years). Only patients with implant-associated osteomyelitis and spinal infections were excluded. Data was collected on demographics, comorbidities, clinical features and diagnostic tests, after consent by the individual patient and with the agreement of the hospital's ethical committee. Grading according to Cierny-Mader, which describes the bone involvement as well as the host status, was done at the time of surgery. ${ }^{5}$ All patients had pre-operative X-rays of the affected limb. These were made with a classic X-ray machine (Philips, Eindhoven, the Netherlands), producing hard-copy images (Figure 1). At followup in 2016, the hospital had acquired a modern digital system (DigiMedX, Medex Loncin SA, Liège, Belgium).

All patients were operated by the two senior authors (JG \& PM). Surgical debridement consisted of thorough bony debridement with removal of all known sequestrae, saucerisation of the hypertrophic cortex until punctate bleeding was observed (paprika sign), lavage with at least $3 \mathrm{~L}$ of Ringers lactate, curettage of all fistula and removal of abscessed soft tissue. Finally, bony defects were filled with bioactive glass granules (Bonalive $\AA$ 1.0-2.0 mm granules, BonAlive Biomaterials Ltd, Turku, Finland) in order to obliterate the dead space. All wounds were closed primarily without need for plastic surgery involvement. Fistulae were curetted but never closed primarily.

During surgery, deep tissue cultures were taken using the standard surgical sampling technique (Oxford protocol: separate instruments for each sample, no-touch technique, minimum of three samples, no suction until samples are taken) $)^{6-8}$ and IV amoxicillin-clavulanic acid started and adjusted to the result of the cultures when they became available. Patients received antibiotics for a total time of two weeks.

Description of the treatment above is identical to our local Dutch protocol, except for the fact that patients receive a total of six weeks of antibiotics. This was, however, not possible due to the restricted financial resources of patients.

After discharge, patients were reviewed at regular intervals and at one and two years post-operatively. The last outpatient review was done in March 2016.

\section{Results}

Nineteen osteomyelitic locations were operated in 18 patients (one patient with humeral and femoral osteomyelitis). Localisation was tibia in ten cases, femur in seven, humerus in two. According to the Cierny-Mader classification, eight were type III (localised) and ten type IV (diffuse). Hosts were ten grade $A$ and eight grade B. There were no exclusion criteria. Five patients were sickle-cell positive. Average preoperative sedimentation rate was $58 \mathrm{~mm} / \mathrm{h}$ (range 9-100 mm/h), and average leucocyte count 7 028/ $\mathrm{mm}^{3}$ (range 5 400$\left.10000 / \mathrm{mm}^{3}\right)$

The volume of the bony defect, filled with bioactive glass granules, was $48 \mathrm{cc}$ on average (range 10-100 cc). Tissue cultures revealed $S$. aureus in six cases, Proteus species in six, S. epidermidis in two, Pseudomonas in one, Enterobacter in one, and no organism was cultured in two (Table 1). Unfortunately, we were not able to get antibiotic sensitivities in all cases, but the majority in which we did, did not

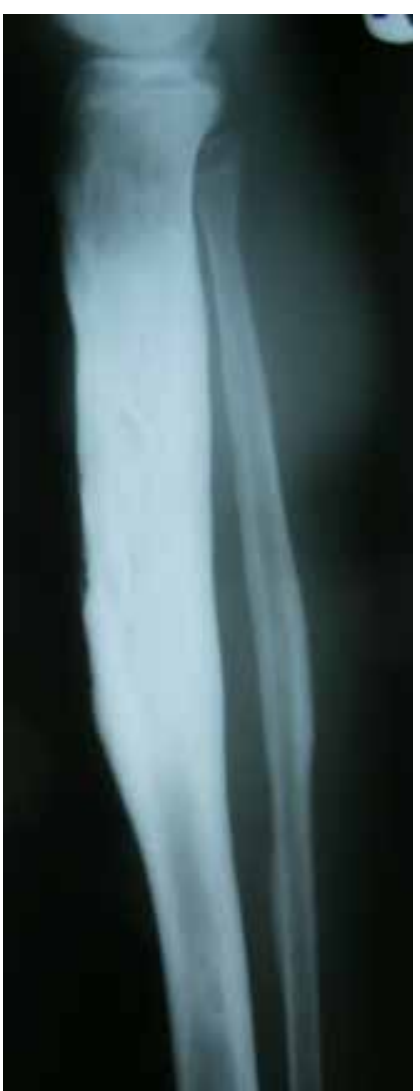

Figure 1. Radiograph of a diffuse suboptimal quality, making the identification of sequestrae very difficult. tibial osteomyelitis. Note the show multi-resistant patterns.

At one-year follow-up, only seven patients (38\%) were able to be assessed in person at the outpatient clinic. Of the other 11, four were able to be contacted by mobile phone. All but one were infectionfree at that time (55\%). The other seven were lost to follow-up. Figure 2 shows a one-year post-operative image of a defect filled with bioactive glass granules. Extra effort was made to see all patients back at the two-year follow-up in March 2016 by reaching out to them in different ways (telephone, mail, community hospitals); we were able to get 13 patients (72\%) back to the clinic and five were lost to follow-up (could not even be contacted by phone).

Recurrence (fistula at other than the operated site, but in the same bone) or persistence of infection occurred in eight of those 13 (61.5\%). Five were still infection-free after two years (38\%).

Table I: Cultured micro-organisms in cohort of 18 patients

\begin{tabular}{|l|c|}
\hline \multicolumn{1}{|c|}{ Micro-organism } & N $(\%)$ \\
\hline Staphylococcus aureus & $6(33 \%)$ \\
\hline Proteus mirabilis & $6(33 \%)$ \\
\hline Staphylococcus epidermidis & $2(11 \%)$ \\
\hline Pseudomonas aeruginosa & $1(5 \%)$ \\
\hline Enterobacter & $1(5 \%)$ \\
\hline No growth & $2(11 \%)$ \\
\hline
\end{tabular}




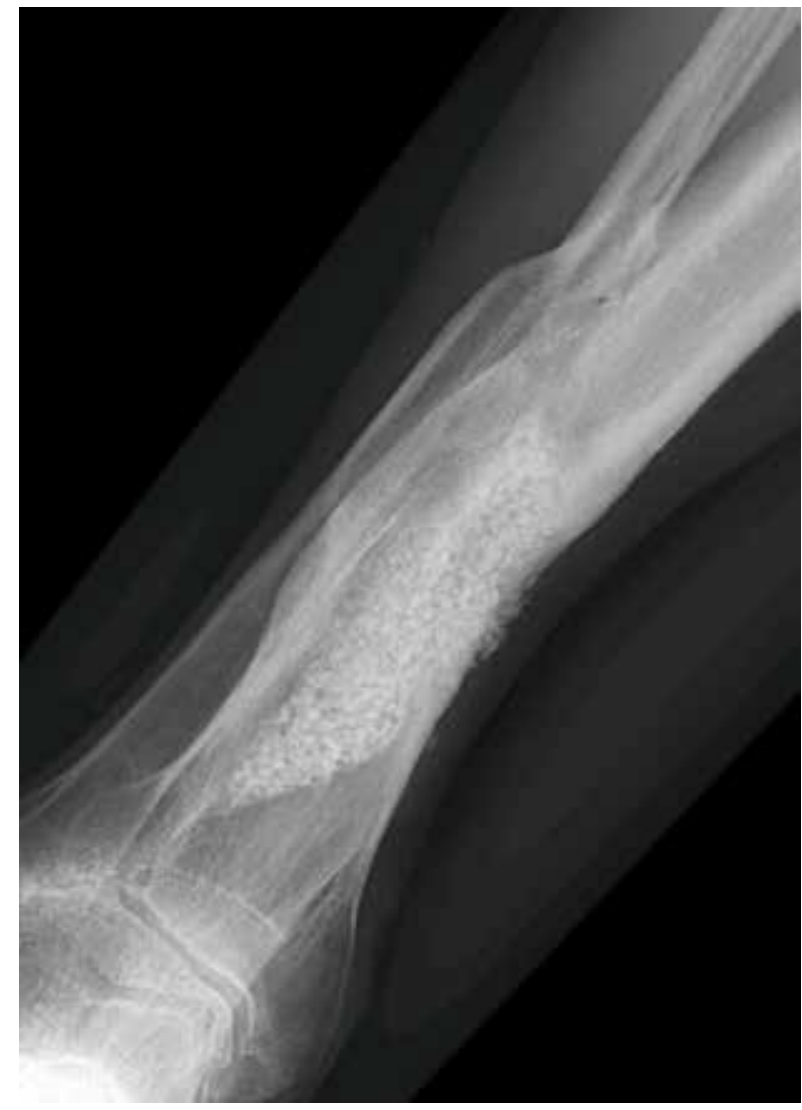

Figure 2. Post-operative (1 y) image showing tibial defect filled with bioactive glass granules. Granules in the soft tissues dissolve over time. Also note improved quality of the PACS image.

\section{Discussion}

Low- and middle-income countries have a high burden of chronic osteomyelitis patients. ${ }^{9-11}$ Often it is the continuation of an acute osteomyelitis in childhood, or the result of open trauma. Predisposing factors are diabetes, sickle cell anaemia and vascular disease., ${ }^{4,12}$

As the affected population is young, hospitalisation is long and lower limbs are the preferred location, chronic osteomyelitis has an important socio-economic impact on the patient and their family. Depending on the state of the health system in these countries, patients often have to pay for the surgery and the medication themselves. This puts an enormous strain on the patient and his or her family and often results in suboptimal treatment (for instance, antibiotic treatment that is not prolonged beyond a couple of days). ${ }^{13,14}$ It is also an important reason why many patients don't seek appropriate medical attention.

Standard surgical treatment includes thorough debridement of all devitalised bone and soft tissue, removal of sequestrae, saucerisation and dead space management. If structural integrity is compromised, the affected limb must be stabilised either externally (external fixation or traction) or with plaster of Paris splinting.

Plain radiographs are of no value in the acute stage of osteomyelitis, but do give information about the extent and presence of sequestrae in later stages. CT scans are superior for identifying sequestrae and MRI for soft tissue involvement, bone marrow abnormalities and evaluation of the extent of the disease..$^{15,16}$ The latter two are more often than not unavailable in the majority of rural and community hospitals in LMICs, thereby complicating work-up before surgery.

Although we attempted to treat these patients in a similar way to how we would in developed countries (by following our own institutional protocol for surgery, microbiology and adjuvant antibiotic therapy), we had significantly more relapses or unsuccessful treatments. Several reasons were identified for why this happened. First, low-quality X-rays, often under- or overexposed, hard-copy films, made accurate pre-operative diagnostic work-up very difficult (evaluation of the extent of the osteomyelitis and the presence of sequestrae) (Figure 1). At the one-year follow-up, the hospital had acquired a digital PACS system which improved the quality of the $X$-rays significantly. In almost all of the relapsed cases, we located other sequestrae, not seen on initial $\mathrm{X}$-rays, that were not removed at the time of initial surgical debridement. This, in itself, was in our opinion one of the main causes of recurrence. Luckily, more and more hospitals are getting access to better imaging, which will undoubtedly improve diagnostic accuracy, although in some developing countries more than half of all rural hospitals still lack basic imaging services. ${ }^{17}$ Scarcity of radiologists and trained medical imaging technologists adds to this problem. ${ }^{18}$ Secondly, a lot of these patients have very longstanding chronic osteomyelitis, due to delayed presentation (the result of lack of transportation but also lack of insight by the patient), treatment by self-proclaimed traditional medicine men and the natural course of chronic osteomyelitis with periods of relative quiescence. ${ }^{19-21}$ This results in enormous cortical hypertrophy and as a consequence in the presence of intracortical abscesses. ${ }^{22,23}$ Saucerisation was done very aggressively, but likely resulted in several of these intracortical abscesses not being removed, again resulting in incomplete eradication of the bony infection. All patients in our study were either Cierny grade III or IV, reflecting the extent and severity of the osteomyelitis. The average size of the bony defect (and thus dead space) to be filled is also significantly higher than what is commonplace in the western world (authors' own experience). Thirdly, access to microbiology is key in adequate antibiotic treatment. We were able to take culture specimens of all patients, but this is not possible in many rural hospitals with limited resources. This can result in inadequate adjuvant antibiotic therapy and persistence of the infection, but also the induction of antibiotic resistance over time.24,25 Also, the duration of the course of antibiotics is generally recommended to be six weeks ${ }^{26,27}$ which is often impossible in LMICs due to financial restraints, thereby also compromising a favourable outcome. ${ }^{27}$ Being financially compromised also influences the possibility of using (often expensive) state-of-the-art biomaterials. In our study, patients received two weeks of antibiotics; longer would have been preferred, but at the time, there was no remuneration scheme in the country, so people had to pay upfront for their own medication, which resulted in patients lacking the required antibiotics. Finally, follow-up is very difficult in LMICs because patients often have to travel long distances and do not come back to the clinic for review, unless some financial or other incentives can be offered to them. If not, some are forced to live with their persisting infection due to poverty, get treated in another hospital, migrate or die. This makes management of postoperative complications and monitoring of medication compliance almost impossible. ${ }^{20,28}$ Often, people can be tracked by phone, but this is often insufficient to assess and monitor the clinical course of the treatment. Also, the high rates of loss to follow-up have the perverse effect that a lot of well-performed studies in these countries will not be published in high level journals.

Other contemporary methods of treating chronic osteomyelitis in a one-stage setting have been described, such as resorbable calcium sulphate pellets loaded with tobramycin (Osteoset ${ }^{\circledR}-\mathrm{T}$, Wright Medical Technology, Memphis, Tennessee, USA). Humm et al. report one recurrence (5\%) of infection in a series of 21 patients with an average follow up of 1.3 years. ${ }^{29}$ Ferguson et al. describe a larger series of 193 patients, followed up for a mean of 3.7 years, with 18 patients suffering from recurrence (9\%). ${ }^{30}$ Most authors describe wound leakage issues with this biomaterial. Unfortunately, no such studies performed in the setting of low-and middle-income countries could be identified. The same applies for the gentamicin-loaded calcium-sulphate/hydroxyapatite bio-composite known as Cerament $\mathrm{G}$ (Bonesupport, Lund, Sweden). Very good results were reported by McNally et al., with a recurrence rate of only $4 \%$ at a mean follow- 
up of 19.5 months, in a setting of one of the top referral centres for osteomyelitis in Europe. ${ }^{31}$ The manufacturer reported on a series of patients treated in the Butare University Teaching Hospital in Rwanda in 2013 with this biomaterial in a press release, but no publication on the follow-up was ever published. Finally, Herafill G, gentamicinloaded calcium sulphate pellets (Heraeus Medical, Werheim, Germany) have been reported to be used in a one-stage setting in Nigeria by Bafor et al. ${ }^{32}$ In their study, 15 patients were treated (46.7\% type III, $13.3 \%$ type IV) resulting in infection eradication in $66.7 \%$ with a mean follow-up of 14.7 months (8-26 months). In this study, no microbiology was performed and no adjuvant antibiotic therapy administered.

Limitations of this study are the high number of patients lost to follow-up, limited and often suboptimal adjuvant antibiotic treatment, and the absence of a control group. We aim to repeat this study in the future as a randomised control trial with adequate follow-up.

\section{Conclusion}

In this paper we conclude that it is currently very difficult to implement state-of-the-art strategies for treating chronic osteomyelitis with modern biomaterials in a setting with often insufficient resources and expect the same outcome. There are a lot of conditions that have to be met, like proper imaging, access to microbiology, availability of adequate antibiotics and follow-up. The concept of treating osteomyelitis in a one-stage setting with modern biomaterials is, however, very attractive in these settings and further research should focus on optimising the implementation thereof, decreasing the need for antibiotic administration and reducing costs in order to offer these treatments to many more patients.

\section{Ethics statement}

Before the commencement of this study, consent was obtained from the individual patients, and with the agreement of the hospital's ethical committee.

\section{References}

1. Bickler SW, Sanno-Duanda B. Epidemiology of paediatric surgical admissions to a government referral hospital in the Gambia. Bulletin of the World Health Organization. 2000;78(11):1330-6.

2. Ozgediz D, Riviello R. The 'other' neglected diseases in global public health: surgical conditions in sub-Saharan Africa. PLoS medicine. 2008;5(6):e121.

3. Museru LM, McHaro CN. Chronic osteomyelitis: a continuing orthopaedic challenge in developing countries. International Orthopaedics. [journal article]. 2001;25(2):127-31.

4. Tice AD, Hoaglund PA, Shoultz DA. Risk factors and treatment outcomes in osteomyelitis. Journal of Antimicrobial Chemotherapy. 2003;51(5):1261-8.

5. Cierny III G, Mader JT, Penninck JJ. The Classic: A Clinical Staging System for Adult Osteomyelitis. Clinical Orthopaedics and Related Research. 2003;414:7-24.

6. Bose D, Kugan R, Stubbs D, McNally M. Management of infected nonunion of the long bones by a multidisciplinary team. Bone \& Joint Journal. 2015;97-B(6):814-7.

7. McNally M, Sendi P. Implant-associated osteomyelitis of long bones. Bone and Joint Infections: John Wiley \& Sons, Ltd; 2015. p. 303-23.

8. Atkins BL, Athanasou N, Deeks JJ, Crook DWM, Simpson H, Peto TEA, et al. Prospective evaluation of criteria for microbiological diagnosis of prosthetic-joint infection at revision arthroplasty. Journal of Clinical Microbiology. 1998;36(10):2932-9.

9. Agaja S, Ayorinde R. Chronic osteomyelitis in Ilorin, Nigeria. S Afr J Surg. 2008;46(4):116-8.

10. Callistus KB, Alhassan A, Majeed SF, Mogre V. Chronic osteomyelitis in a Ghanaian Specialist Hospital. Advances in Applied Science Research. 2015:6(2):150-3.
11. Atijosan $O$, Rischewski $D$, Simms $V$, Kuper $H$, Linganwa $B$, Nuhi $A$, et al. A national survey of musculoskeletal impairment in Rwanda: prevalence, causes and service implications. PLoS One. 2008;3(7):e2851.

12. Wald ER. Risk factors for osteomyelitis. American Journal of Medicine. 1985;78(6):206-12.

13. Stanley CM, Rutherford GW, Morshed S, Coughlin RR, Beyeza T. Estimating the healthcare burden of osteomyelitis in Uganda. Transactions of the Royal Society of Tropical Medicine and Hygiene. 2010;104(2):139-42.

14. Gosselin RA. The increasing burden of injuries in developing countries: direct and indirect consequences. Techniques in Orthopaedics. 2009;24(4):230-2

15. Pineda C, Espinosa R, Pena A. Radiographic imaging in osteomyelitis: the role of plain radiography, computed tomography, ultrasonography, magnetic resonance imaging, and scintigraphy. Seminars in Plastic Surgery. 2009;23(2):80-9.

16. Lee YJ, Sadigh S, Mankad K, Kapse N, Rajeswaran G. The imaging of osteomyelitis. Quantitative Imaging in Medicine and Surgery. 2016;6(2):184-98

17. Henry JA, Windapo O, Kushner AL, Groen RS, Nwomeh BC. A survey of surgical capacity in rural southern Nigeria: Opportunities for change. World Journal of Surgery. 2012;36(12):2811-8.

18. Everton KL, Mazal J, Mollura DJ. White Paper report of the 2011 RAD-AID Conference on International Radiology for Developing Countries: Integrating Multidisciplinary Strategies for Imaging Services in the Developing World. Journal of the American College of Radiology. 2012;9(7):488-94.

19. Joshipura MK, Shah HS, Patel PR, Divatia PA, Desai PM. Trauma care systems in India. Injury. 2003;34(9):686-92.

20. Grimes CE, Bowman KG, Dodgion CM, Lavy CBD. Systematic review of barriers to surgical care in low-income and middle-income countries. World Journal of Surgery. 2011;35(5):941-50.

21. Ciampolini J, Harding KG. Pathophysiology of chronic bacterial osteomyelitis. Why do antibiotics fail so often? Postgraduate Medical Journal. 2000;76(898):479-83.

22. Scott ML, Robert EL, Catherine NP. Cortical lesions of the tibia: characteristic appearances at conventional radiography. RadioGraphics. 2003;23(1):157-77.

23. Gold RH, Hawkins RA, Katz RD. Bacterial osteomyelitis: findings on plain radiography, CT, MR, and scintigraphy. American Journal of Roentgenology. 1991 2017;157(2):365-70.

24. Van Boeckel TP, Gandra S, Ashok A, Caudron Q, Grenfell BT, Levin SA, et al. Global antibiotic consumption 2000 to 2010: an analysis of national pharmaceutical sales data. The Lancet Infectious Diseases. 2017;14(8):742-50.

25. Ashley EA, Lubell $Y$, White NJ, Turner P. Antimicrobial susceptibility of bacterial isolates from community acquired infections in Sub-Saharan Africa and Asian low and middle income countries. Tropical Medicine \& International Health. 2011;16(9):1167-79.

26. Calhoun JH, Manring MM. Adult osteomyelitis. Infectious Disease Clinics. 2005;19(4):765-86.

27. Lazzarini L, Lipsky BA, Mader JT. Antibiotic treatment of osteomyelitis: what have we learned from 30 years of clinical trials? International Journal of Infectious Diseases. 2005;9(3):127-38.

28. Ologunde R, Rufai SR. Surgical follow-up in low-income and middleincome countries. The Lancet Global Health. 2013;1(3):e132.

29. Humm G, Noor S, Bridgeman P, David M, Bose D. Adjuvant treatment of chronic osteomyelitis of the tibia following exogenous trauma using OSTEOSET®-T: a review of 21 patients in a regional trauma centre. Strategies in Trauma and Limb Reconstruction. 2014;9(3):157-61.

30. Ferguson JY, Dudareva M, Riley ND, Stubbs D, Atkins BL, McNally MA. The use of a biodegradable antibiotic-loaded calcium sulphate carrier containing tobramycin for the treatment of chronic osteomyelitis. Bone Joint J. 2014;96(6):829-36.

31. McNally MA, Ferguson JY, Lau ACK, Diefenbeck M, Scarborough $M$, Ramsden AJ, et al. Single-stage treatment of chronic osteomyelitis with a new absorbable, gentamicin-loaded, calcium sulphate/hydroxyapatite biocomposite. Bone Joint J. 2016;98(9):1289-96.

32. Bafor A, Akpojevwe E. One stage treatment of chronic osteomyelitis using antibiotic impregnated bone graft substitute: a preliminary report. Annals of Biomedical Sciences. 2017;16(1):1-9. 